\title{
Quality Prediction Model Based on Variable-Learning-Rate Neural Networks in Tobacco Redrying Process
}

\author{
Jiankang Yin ${ }^{1,2, a}$, Changhua Chen ${ }^{3, b(*)}$ \\ ${ }^{1}$ School of Economics \& Management, South West Jiao Tong University, Chengdu 610031, China \\ ${ }^{2}$ Sichuan Provincial Tobacco Companies Chengdu Company, Chengdu 610072, China \\ ${ }^{3}$ School of Business Administration, Xihua University, Chengdu 610039, China \\ a673327681@qq.com, bcch.scu@163.com
}

Keywords: Tobacco; Drying Process; Back Propagation Neural Network; Variable Learning Rate; Quality Prediction Model

Abstract. This paper presents an innovative method with the variable-learning-rate-based back propagation neural network (BPNN) for establishing a quality prediction model of tobacco redrying process. First, characteristics of the process and correlation of the process variables are analyzed, and eight input parameters and two output quality indicators of the model are determined. Second, a quality prediction model of the tobacco redrying process is established by using the BPNN structure. In the process of network training, BP algorithm is improved by using the method of variable learning rate, and satisfactory prediction results are obtained. Finally, in order to verify the effectiveness of this method, the improved BPNN model is applied for simulation experiment, and is compared with ordinary BPNN. The prediction results show that the improved model possesses strong self-learning function and higher prediction accuracy.

\section{Introduction}

Tobacco redrying is a re-curing process after the tobacco drying for the first time, which has a direct impact on the production quality of the cigarette industry. This process includes three phases: drying, cooling and moisture regain [1]. Quality control is one of the research emphasis during tobacco redrying process, and the method of intelligent control is usually applied to the quality control of tobacco redryer to realize that the parameters of every PID control link of tobacco redryer, it automatically adjusted on line, and the output tobacco moisture and temperature is controlled at the range of technology demand [2]. At present, predictive control is a good method to guarantee the quality of products, and the establishment of a quality prediction model is the premise to realize the predictive control.

The tobacco redrying process has obvious characteristics of multi-interference, strong coupling, great hysteresis, nonlinear and uncertainty. The neural network method is more effective in the establishment of the quality prediction model of tobacco redrying process. The neural network method can describe the characteristics of the object without establishing the physical model of the object, it has the advantages of parallel processing, strong learning ability and sound robustness. Therefore, it has been widely used in complex systems [3, 4]. In recent years, scholars have put forward the neural network method to apply in object of industrial production modeling, which has achieved significant control effect [5-9]. However, this method is less applied in the modeling of tobacco redrying process.

In this research, we developed a variable-learning-rate neural network based quality prediction model for the tobacco redrying process.

\section{Characteristic Analysis of Process}

Impact factors of Process. In the tobacco redrying process, the most direct quality indicators are tobacco moisture and temperature. The technical index requires that tobacco moisture is controlled between $11.5 \%$ and $13.5 \%$, and tobacco temperature is controlled between $50{ }^{\circ} \mathrm{C}$ and $60{ }^{\circ} \mathrm{C}$. The goal 
of the production process control is that process parameters are adjusted to make the tobacco moisture and temperature reach the process index requirements.

Assuming the external environment of tobacco redrying is the same, and for tobacco of the same planting area, the same variety and the same grade, the main influence factors of the tobacco moisture and temperature are: tobacco leaf moisture at entrance $\left(M_{e}\right)$, tobacco temperature at entrance $\left(T_{e}\right)$, tobacco feed flow $\left(F_{f}\right)$, the speed of tobacco transport chain car $(V)$, temperature of four drying sections ( $\left.T_{d 1}, T_{d 2}, T_{d 3}, T_{d 4}\right)$, heating steam $\left(S_{h}\right)$, moisture of cooling zone $\left(M_{c z}\right)$, temperature of cooling $\operatorname{zone}\left(T_{c z}\right)$, water flow of moisture regain $\left(F_{r}\right)$, steam of moisture regain $\left(\mathrm{S}_{r}\right)$ and so on, and there exists strong coupling between these factors.

Correlation analysis. 500 groups samples of data are obtained randomly from the historical database of the same planting area, the same variety and the same grade. This includes 13 data samples: $M_{e}, T_{e}, F_{f}, V, T_{d 1}, T_{d 2}, T_{d 3}, T_{d 4}, S_{h}, M_{c z}, T_{c z}, F_{r}, \mathrm{~S}_{r}$. Those samples are used for statistical analysis. The correlation between any two variables can be calculated by Eq. 1 [9]:

$$
r=\frac{\sum X Y-\frac{\sum X \sum Y}{N}}{\sqrt{\left(\sum X^{2}-\frac{\left(\sum X\right)^{2}}{N}\right)\left(\sum Y^{2}-\frac{\left(\sum Y\right)^{2}}{N}\right)}}
$$

In the Eq. 1, $r$ represents correlation coefficient, $X$ and $Y$ represent any two variables, and $N$ represents number of samples. When the correlation coefficient of two variables $r=1$, it shows that the two variables are completely correled; when $r=0$, it shows that the two variables do not have linear correlation; when $r>0.7$, it could be considered that strong correlation exists between the two variables. According to the strong correlation coefficient standard 0.7, there is a strong correlation between $S_{h}$ and $T_{d 1}, T_{d 2}, T_{d 3}, T_{d 4}$. Similarly, it can draw that there exists a strong correlation between $S_{h}$ and $M_{c z}, S_{h}$ and $T_{c z}, T_{d 1}$ and $T_{c z}, T_{d 2}$ and $T_{c z}, T_{d 3}$ and $T_{c z}, T_{d 4}$ and $T_{c z}$. To ensure that the variables of neural network input layer have mutual independence, this paper chooses $M_{e}, T_{e}, F_{f}, V, M_{c z}, T_{c z}, F_{r}$ and $S_{r}$ as the input parameters of neural network model.

\section{Neural Network Prediction Model of Tobacco Redrying Process}

BPNN is a mature neural network. The BP algorithm is essentially a gradient descent method, and the learning results exist the inevitable problem of local minimum. As the learning rate remains unchanged in the learning process, the algorithm convergence speed may be slow, or even non-convergent. Therefore, in order to improve training speed and model accuracy, the ordinary BP algorithm must be improved. We adopted a method of variable learning rate based.

Quality prediction model. BPNN with 3-layers is adopted to establish a prediction model. The inputs of the neural network are determined to: $M_{e}, T_{e}, F_{f}, V, M_{c z}, T_{c z}, F_{r}$ and $S_{r}$, and the model outputs are tobacco moisture and temperature of re-dryer exit (respectively as $M$ and $T$ ). So the number of neuron input and output are 8 and 2. And the hidden layer neuron number is calculated to 20 according to the empirical formula. The neural network structure is shown in Fig. 1.

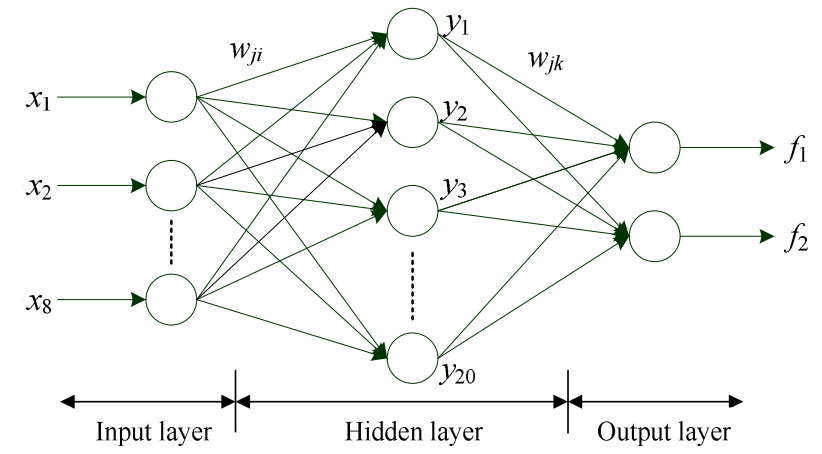

Fig. 1. Tobacco leaves drying moisture neural network model structure diagram 
In Fig. $1, x_{i}(i=1,2, \ldots, 8)$ is input layer variables, $y_{j}(j=1,2, \ldots, 20)$ is hidden layer variables, $f_{k}(k=1$, 2 ) is output layer variables, $w_{j i}$ is the weight value from the $i$ th neurons of input layer to the $j$ th neurons of hidden layer, $w_{j k}$ is the weight value from the $j$ th neurons of hidden layer to the $k$ th neurons of output layer. By the sigmoid-tansig non-linear activation function, the neural network structure of the tobacco redrying process export moisture and temperature prediction model was determined as:

$$
f_{k}=(M, T)^{\mathrm{T}}=\sum_{j=1}^{20} w_{j k} \tan \operatorname{sig}\left(\sum_{i=1}^{8} w_{j i} x_{i}+b_{j}\right)+b_{k}
$$

In Eq. 2, $w_{j i}$ and $w_{j k}$ represent the weight value, $b_{j}$ represents the corresponding threshold value of the $j$ th neurons of hidden layer, and $b_{k}$ represents the corresponding threshold value of the $k$ th neurons of output layer. The weight value $w_{j i}, w_{j k}$ and threshold value $b_{j}, b_{\mathrm{k}}$ were obtained from the training of BPNN.

BP algorithm of variable learning rate. The prediction model is the basis of parameter optimization, whose accuracy has great significance to ensure optimization effectiveness and reliability. Although the ordinary BP algorithm is effective, the convergence speed is very slow near the target point. When describing complex systems, it can never ensure the global convergence and falls into the local minimum. Thereby the ordinary BP algorithm needs to be improved. There are mainly two ways to improve the BP algorithm; using a heuristic learning method and using a more efficient optimization algorithm. Usually, two optimization algorithms of increasing momentum terms and changing learning rate are adopted to improve network training performance $[9,10]$. This paper uses a variable-learning-rate-based BP algorithm training Eq. 2.

Through the adjustment of network weight value $w$ to the error function decrease along the negative gradient direction:

$$
w(k+1)=w(k)-\eta(t) \frac{\partial E}{\partial w(k)}
$$

In Eq. 3, $E=\frac{1}{N} \sum_{k=1}^{N}\left[x_{0}(k)-\hat{x}_{0}(k)\right]^{2}, N$ is learning sample number, $x_{0}(k) 、 \hat{x}_{0}(k)$ are network output value and expectation value of the $k$ th sample. The goal of network training is to minimize $E$ through adjusting weight value and threshold value constantly.

When adjusting network weight value, it assumes that learning rate $\eta$ is a linear function of the number of iterative learning (expressed as $t$ ), the maximum learning rate is $\eta_{\max }$, and the minimum learning rate is $\eta_{\min }$. After each learning, the learning rate variable quantity is defined to:

$$
\Delta \eta=\left(\eta_{\max }-\eta_{\min }\right) / t_{\max }
$$

Then the learning rate of the $t$ th time learning is:

$$
\eta(t)=\eta_{\max }-\Delta \eta \times t=\eta_{\max }-\left(\eta_{\max }-\eta_{\min }\right) \times t / t_{\max }
$$

In the equation, $t_{\max }$ and $\eta$ are obtained empirically. Through experimentation, when $\eta \in[0.1,0.9]$ better results can be achieved.

Network Learning. The steps of improving BP network training are: (1) Initializing weights and thresholds. Giving the initial value of weights and thresholds between -1 and 1 at Random, and initial raw data of input and output. (2) Providing a learning sample of network training. (3) According to Eq. 2, calculate the actual output of the network. (4) Comparing the current training error and the last time training error, and adjust weight according to Eq. 3. (5) Judging whether the error meets the scheduled accuracy indexes. If satisfied, the training ends, then take the determined weight and threshold value into Eq. 2, and output prediction results. Otherwise return to step(2). 


\section{Results and discussion}

This research presented a variablelearningratebased approach to create a tobacco redrying process quality predictor. Eight BPNN manufacturing process parameters and two quality indicators were dedicated to train and test the network.

The neural networks were first trained using 450 samples of training data, then 50 samples of verifying data were used to make predictions, and the network performance was obtained by calculating the Root Mean Square Error (RMSE). The BPNN performance is shown in Table 1.

Table 1 Comparison of the network performance between the improved BPNN and ordinary BPNN

\begin{tabular}{|c|c|c|c|}
\hline \multicolumn{2}{|c|}{ Item } & Improved BPNN & Ordinary BPNN \\
\hline \multirow{2}{*}{ Moisture predictor } & Training RMSE & 0.020 & 0.045 \\
\hline & Testing RMSE & 0.012 & 0.027 \\
\hline \multirow{2}{*}{ Temperature predictor } & Training RMSE & 0.0076 & 0.010 \\
\hline & Testing RMSE & 0.0066 & 0.017 \\
\hline
\end{tabular}

It can be seen from Table 1 that the training precision of the improved BPNN moisture and temperature quality predictor had an RMSE of up to 0.020 and 0.0076 , and its testing precision amount was an RMSE of up to 0.012 and 0.0066 . Whereas the training precision of the ordinary BPNN moisture and temperature quality predictor had an RMSE of 0.045 and 0.010 , and its testing precision amount was an RMSE of 0.027 and 0.017. As such, the performance of the improved BPNN quality predictor was better than that of the ordinary BPNN quality predictor.

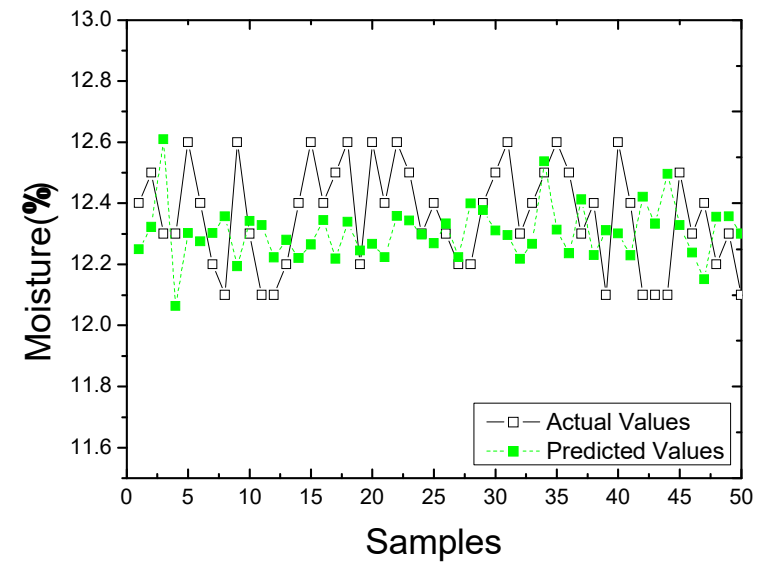

(a) Ordinary BPNN

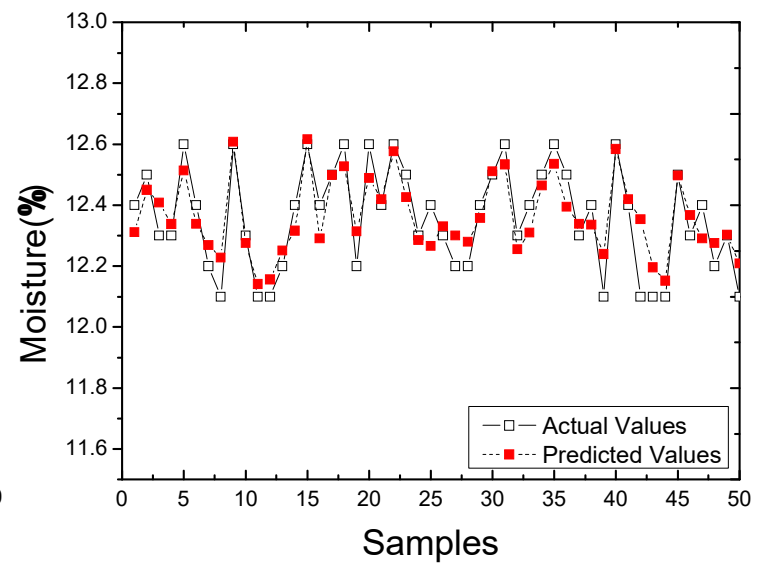

(b) Improved BPNN

Fig. 2. Comparison between the predicted and actual moisture $(\%, w b)$ from (a) Ordinary BPNN and (b) Improved BPNN

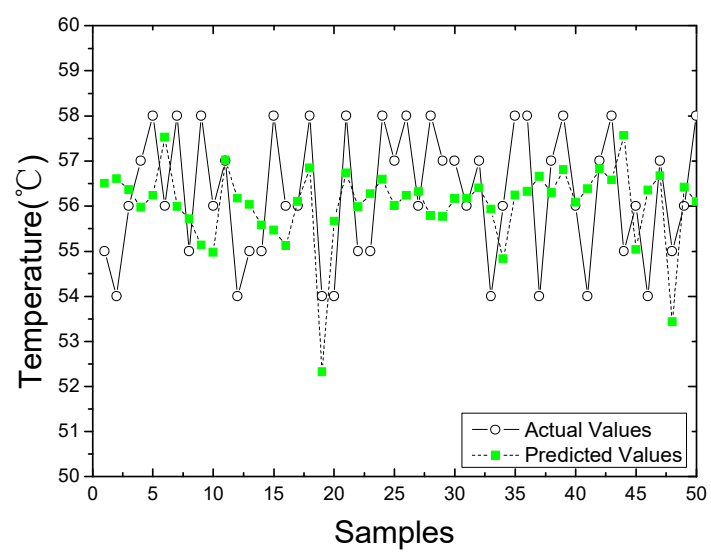

(a) Ordinary BPNN

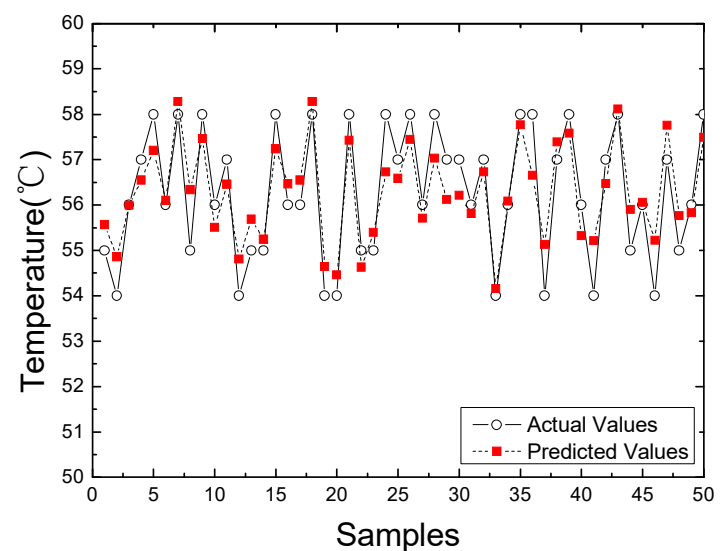

(b) Improved BPNN

Fig. 3. Comparison between the predicted and actual temperature $\left({ }^{\circ} \mathrm{C}\right)$ from (a) Ordinary BPNN and

(b) Improved BPNN 
A comparison between the actual and predicted values via ordinary BPNN and improved BPNN moisture and temperature quality predictors are represented in Fig. 2 and Fig. 3. The results showed that the variable-learning-rate-based BPNN had a better prediction effect than ordinary BPNN, the forecasting accuracy reached more than $90 \%$, and can accurately reflect the quality trend of tobacco redrying machine exports moisture and temperature in the normal production process. Based on these results, improved BPNN can provide effective guidance for solving the tobacco redrying machine quality optimal control problem.

However, because the actual tobacco production process is dynamic, the tobacco redrying process quality prediction BPNN model needs to change constantly as the samples are updated. When the model is undertaking self-learning, the latest samples are joined into the self-learning library files, and the similar and earliest samples are eliminated, so as to always keep a uniform quantity of learning samples. Therefore, in order to enhance the prediction precision of the product quality in the dynamic system, it is essential to extract the dynamic data of the process parameters.

\section{Conclusion}

The tobacco redrying process control and optimal parameter settings for the product properties requires very accurate quality prediction. This research presented a model based on variable learning rate BPNN for quality prediction of the tobacco redrying process. Eight manufacturing process parameters and two quality indicators were dedicated to train and test the networks. In addition, another ordinary BPNN model was employed for comparison. The results revealed that the improved BPNN model proposed not only increased the prediction performance of product quality effectively and obtained more reliable product quality in advance, but also solved the problem of quality modeling difficulties of the tobacco redrying process. In future extensions, the proposed approach will be employed to the process control for improving the prediction precision of the product quality in the tobacco redrying machine.

\section{Acknowledgements}

This work was financially supported by the National Natural Science Foundation of China (51275554) and Xihua University Key project (zw1311543).

\section{References}

[1] Krijnen Henk, Morris Philip: Control Solutions International Vol. 76 (2003), p. 43

[2] Pakowski, Z.; Krupinska, B.; Adamski, R: Inzynieria Chemiczna I Procesowa Vol. 27 (2006), p.507-517

[3] Wilson, J.A.; Zorzetto, L.R.M.: Computer Chemistry Engineering Vol. 21 (1997), p.951-963.

[4] Hagan, M.T.; Demuth, H.B.; Beale, M.H. in: Neural Network Design. Boston, edtied by PWS Publishing, USA (1996)

[5] Yang, C.H.; Deconinck, G. et al.: IEEE Transactions on Neural Networks Vol. 13 (2002), p.229-236

[6] Wang Haifeng, Cao Yunpeng: Parallel Computing Vol. 44 (2015), p.18-36

[7] Li Jun,Chen Jiawen,Liao Weili,Gao Chuanchang: Transactions of the Chinese Society of Agricultural Engineering (Transactions of the CSAE) Vol. 32(2016), p.47-53

[8] Liu Y M, Zhou H F. : Systems Engineering - Theory \& Practice Vol. 36 (2016), p.1890-1897

[9] Xu, C.H.; Wu, M. : Journal of System Simulation Vol. 21(2009), p.1024-1028

[10] Jacobs, R.A.: Neural Networks Vol. 1 (1988), p.295-307 